\title{
PERCEPCIÓN DEL CIRUJANO-DENTISTA RESPECTO DEL USO DEL CONSENTIMIENTO INFORMADO EN EL TRATAMIENTO ODONTOLÓGICO
}

\author{
Cléa Adas Saliba Garbin*, Artênio José Isper Garbin**, \\ Cleide dos Anjos Santos***, Patrícia Elaine Gonçalves****
}

\begin{abstract}
Resumen: Este estudio tuvo por objetivo evaluar: el perfil del cirujano-dentista que realiza curso de especialización en la Facultad de Odontología de Araçatuba/UNESP, Brasil, si éste solicita al paciente y/o a su representante legal el consentimiento informado antes de la realización de los procedimientos, y si decide con el paciente sobre su tratamiento. Se elaboró, validó y aplicó un cuestionario, y los datos obtenidos fueron analizados por medio del programa EPI-INFO 3.3. Se concluyó que, dado que aún hay cirujano-dentistas que antes de empezar un tratamiento no obtienen ningún tipo de documento, una gran parte de ellos está totalmente vulnerable frente a un proceso ético o jurídico y que es necesario tomar conciencia sobre estos aspectos.
\end{abstract}

Palabras clave: consentimiento informado, relaciones dentista-paciente, odontología, bioética

\section{PERCEPTION OF THE SURGEON-DENTIST REGARDING THE USE OF INFORMED CONSENT IN DENTAL TREATMENT}

\begin{abstract}
The objective of this study was to evaluate: the profile of the surgeon-dentist that undergoes a course in specialization at the Odontology Faculty of Aracatuba/UNESP, Brazil; if they requested informed consent from the patient and/or their legal representative before the realization of procedures, and if the treatment was decided upon with the patient. It was elaborated, validated and applied in questionnaire form, and the data obtained was analyzed by the program EPI-INFO 3.3 The conclusion was, given that surgeon-dentists still do not obtain any type of document before beginning treatment, a large percentage of them are totally vulnerable if submitted to ethical or legal prosecution and that it is necessary to be aware of this aspect.
\end{abstract}

Key words: Informed consent, dentist-patient relations, odontology, bioethics

\section{PERCEPÇÃO DO CIRURGIÃO DENTISTA A RESPEITO DO USO DO CONSENTIMENTO INFORMADO NO TRATAMENTO ODONTOLÓGICO}

Resumo: Este estudo, teve como objetivo avaliar o perfil do cirurgião dentista que realiza curso de especializaçáo na Faculdade de Odontologia de Araçatuba/ UNESP, Brasil, se este solicita ao paciente ou a seu representante legal o consentimento informado antes da realização dos procedimentos, e se decide com o paciente a respeito de seu tratamento. Elaborou-se um questionário, validou-se e se aplicou o questionário e os dados obtidos foram analisados pelo programa EPI-INFO 3.3. Conluiu-se que, dado que ainda existem cirurgióes dentistas que antes de implementar um tratamento não obtem nenhum tipo de documento. Uma grande parte deles está totalmente vulverável frente a um processo ético ou jurídico e que é necesario tomar conciëncia sobre estes aspectos.

Palavras chave: consentimento informado, relações dentista-paciente, odontologia, bioética

\footnotetext{
Profesora Adjunta, Departamento de Odontología Infantil y Social, Facultad de Odontología de Araçatuba, Universidad Estadual Paulista - UNESP, Brasil

** Profesor Doctor, Departamento de Odontología Infantil y Social, Facultad de Odontología de Araçatuba, Universidad Estadual Paulista - UNESP, Brasil

*** Alumna Facultad de Odontología de Araçatuba, Universidad Estadual Paulista - UNESP, Brasil

**** Alumna Programa de Posgrado en Odontología Preventiva y Social, Facultad de Odontología de Araçatuba, Universidad Estadual Paulista - UNESP, Brasil

Correspondencia: cgarbin@foa.unesp.br
} 


\section{Introducción}

El consentimiento se relaciona con el principio de la inviolabilidad del cuerpo humano. La primera vez que el término "consentimiento informado" fue utilizado en una sentencia judicial fue en el caso Salgo vs. Leland Stanford Jr., University Board of Toistees, 1997, California(1). Es un procedimiento de gran importancia en la investigación que involucra seres humanos, pues en éste queda establecida la voluntariedad de la participación de los sujetos de la investigación(2). Sin embargo, su importancia no se restringe a la investigación científica, sino que alcanza también la práctica clínica, enfatizando la importancia de la ética y la moral en los consultorios odontológicos(3,4). Farah y Ferraro(5) afirmaron que el consentimiento del paciente, antes del inicio de cualquier procedimiento realizado por el cirujano dentista, es una condición que debe ser respetada, observándose el principio de la autonomía, por el cual todos tienen el derecho de ser autores de su propio destino y decidir qué desean en su vida(6).

Cuando es obtenido de modo correcto, el consentimiento informado legitima y fundamenta la práctica clínica o de investigación como justa y éticamente correcta(7). Su no utilización torna vulnerables a los profesionales en diversos procesos judiciales, aumentando cada vez más los procesos contra cirujanos dentistas. Generalmente, esas acciones están relacionadas con la insatisfacción del paciente respecto del tratamiento realizado, error de tratamiento o carencia de información, entre otros, infringiendo las legislaciones vigentes.

El aumento en las demandas trae aparejados la toma de conciencia de la población respecto de sus derechos como consumidora; el advenimiento del Código de Defensa del Consumidor Brasileño en los años 90; la pérdida del poder adquisitivo de la clase media, que se volvió más rigorosa y exigente respecto de la calidad de los servicios, y la saturación del mercado de trabajo. Ello lleva a algunos profesionales a la competencia desleal, comprometiendo así sus servicios prestados $(8,9)$.

El presente estudio tuvo por objetivo evaluar el perfil del cirujano dentista que realiza curso de especialización en la Facultad de Odontología de Araçatuba/UNESP, Brasil, si éste solicita al paciente y/o a su representante legal el consentimiento informado antes de la realización de los procedimientos, y si decide con el paciente sobre su tratamiento.

\section{Metodología}

Se trató de una investigación transversal y cuantitativa, que incluyó a todos los cirujanos dentistas que realizaban el curso de especialización en la Facultad de Odontología de Araçatuba/UNESP y que accedieron a participar en la investigación.

El proyecto fue sometido a la evaluación del Comité de Ética en Investigación de la Facultad de Odontología de Araçatuba FOA/UNESP, bajo proceso número 2006-00071.

De acuerdo con las reglas relacionadas con la ética en investigación con seres humanos, los participantes fueron informados sobre la voluntariedad de la participación y la mantención del secreto de sus identidades. Se respetaron las reglas de la Resolución $\mathrm{N}^{\circ} 196$, de 10 de octubre de 1996, del Consejo Nacional de la Salud/Ministerio de Salud Brasileńo y sus resoluciones complementarias.

Para la tabulación de los datos fue confeccionado un banco de datos, utilizándose el programa EPINFO 3.3, de libre distribución por la Organización Mundial de la Salud (OMS). Para la incorporación de los datos de cada respuesta se creó un número de identificación que representa un campo en ese banco de datos(10). Por medio de este último se puede realizar el levantamiento de frecuencia de los datos y la confección de gráficos y tablas que explican mejor las respuestas obtenidas.

\section{Resultados}

Se entregaron 95 cuestionarios, distribuidos en las especialidades ofrecidas en la Facultad de Odontología de Araçatuba: Operatoria Dental, Endodoncia, Implantodoncia, Odontología Pediátrica, Odontología Preventiva y Social, Ortodoncia, Periodoncia y Prótesis Dental, de acuerdo con la cantidad de alumnos en sus respectivas especialidades. Se obtuvo respuesta del 85,9\% de ellos.

Entre los entrevistados, el 29,4\% son hombres y el 70,6\% mujeres, y un $63,5 \%$ presentan hasta cinco años de profesión. Sobre el tipo de sistema en que trabajan esos profesionales, el 49,4\% dice ser autónomo, un 11,8\% trabaja por porcentaje y un 5,9\% recibe sueldo fijo.

Sobre la presentación por los cirujanos dentistas de las alternativas de tratamiento al paciente (gráfico 1), se observó que el $83,5 \%$ alega que informan todas las alternativas, mientras que el $12,9 \%$ presenta sólo aquellas en las que tienen dominio. 
Percepción del cirujano-dentista respecto del uso del consentimiento informado en el tratamiento odontológico - Cléa Adas Saliba Garbin, Artênio José Isper Garbin, Cleide dos Anjos Santos, Patrícia Elaine Gonçalves

GRÁFICO 1- Distribución porcentual de la opinión del cirujano dentista sobre la presentación de todas las alternativas de tratamiento, cuando el caso posee más de una opción. Araçatuba - SP. 2007.

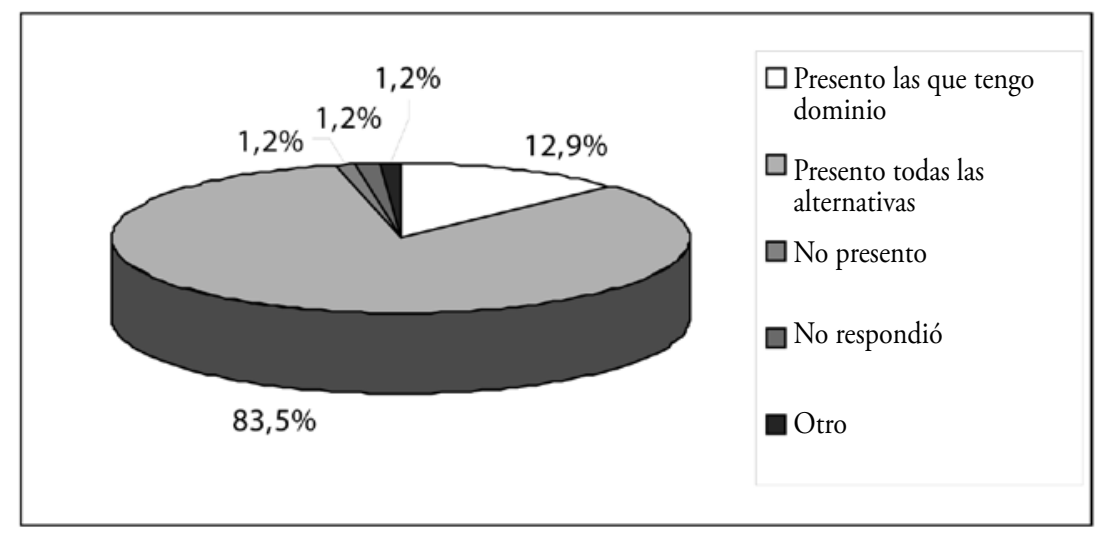

Respecto de la decisión sobre el tratamiento odontológico (gráfico 2), un 89,4\% menciona que debe ser realizada de común acuerdo entre el profesional y el paciente. Sin embargo, el 29\% de los investigados relata

que la participación del paciente y/o su responsable legal en la decisión de tratamiento interfiere de manera negativa, pues éste puede optar por el tratamiento que el cirujano dentista juzga no ser el mejor (gráfico 3).

GRÁFICO 2 - Distribución porcentual de la opinión del cirujano dentista sobre la decisión del tratamiento odontológico. Araçatuba SP. 2007.

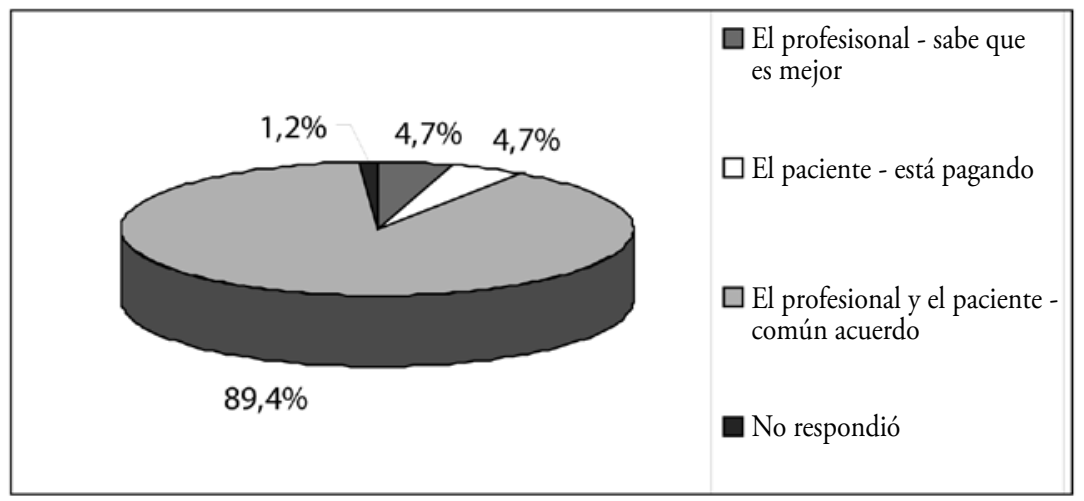

GRÁFICO 3 - Distribución porcentual de la opinión del cirujano dentista sobre la participación del paciente y/o representante legal en la decisión de tratamiento. Araçatuba - SP. 2007.

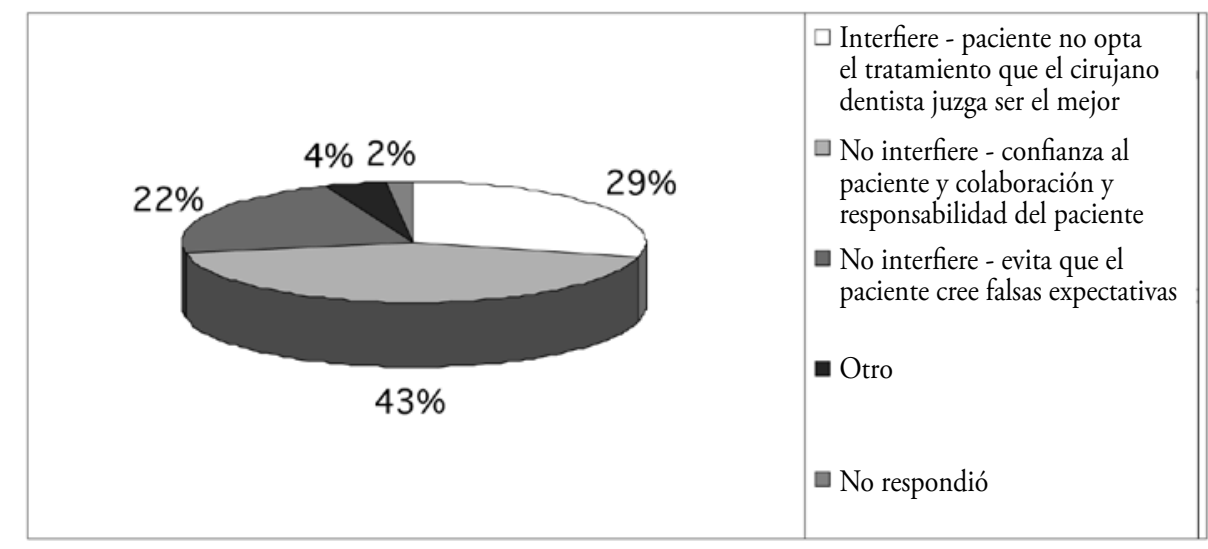


Acerca del lenguaje empleado por el profesional en el tratamiento, el 44\% (gráfico 4) hace uso del lenguaje técnico, explicando apenas las dudas del paciente, $y$ un $48 \%$ emplea lenguaje común para que el paciente tenga el entendimiento sobre su tratamiento.

GRÁFICO 4- Distribución porcentual de la opinión del cirujano dentista cuanto el lenguaje utilizado en el tratamiento odontológico. Araçatuba - SP. 2007.

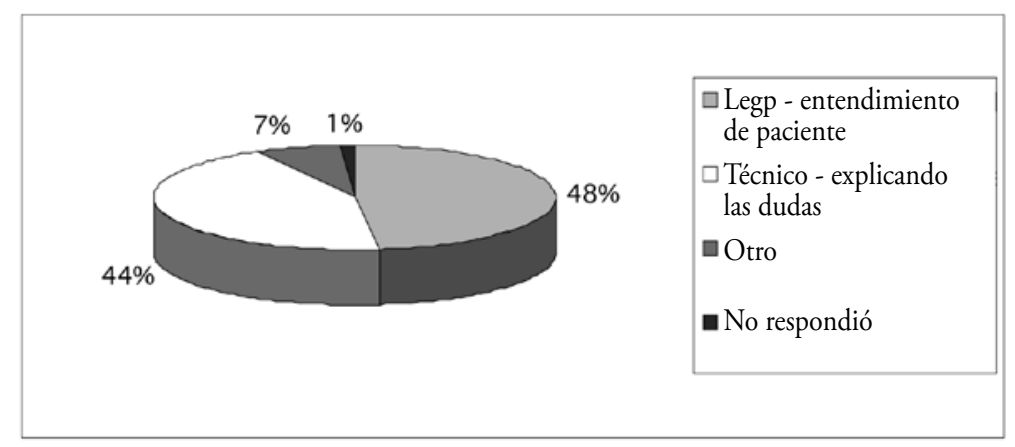

Con relación al documento solicitado por el profesional al paciente antes del tratamiento, se observó que más de la mitad de la población evaluada (54\%) obtiene la autorización para el tratamiento y apenas el 25\% solicita el consentimiento informado. Sin embargo, se debe resaltar que $9 \%$ de los investigados está totalmente vulnerable ante un proceso ético o jurídico, porque no solicitan ninguna prueba ético-legal al paciente antes del tratamiento (gráfico 5).

GRÁFICO 5- Distribución porcentual de la opinión del cirujano dentista sobre cuál prueba ético-legal debe ser solicitada al paciente antes del tratamiento odontológico. Araçatuba - SP.2007.

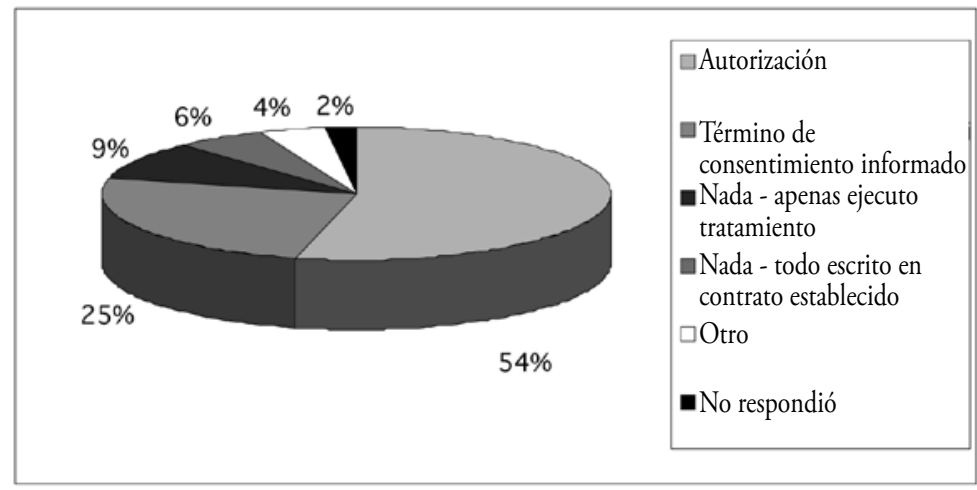

\section{Discusión}

La percepción del paciente como un todo, con personalidad específica, problemas y anhelos particulares es fundamental para la buena comunicación y el éxito del tratamiento odontológico(11). Ante eso, los cirujanos dentistas de las más variadas especialidades han procurado asumir una postura más humana frente a sus pacientes, mejorando la calidad del servicio prestado en función de todo el avance técnico-científico en la actualidad.
En lo que se refiere a la presentación de las alternativas de tratamiento al paciente, se observó que un 12,9\% de los cirujanos presentan sólo aquellas en las que tienen dominio, mientras que un $83,5 \%$ informan todas las alternativas para el determinado caso, lo que está de acuerdo con la opinión de Munhoz et al.(12) y Chiccone(13), pues el paciente tiene el derecho moral de ser informado sobre la naturaleza y los objetivos de los procedimientos diagnósticos, preventivos o terapéuticos; de su confidencialidad, de la duración de los tratamientos, de los beneficios, probables incomodidades y riesgos 
Percepción del cirujano-dentista respecto del uso del consentimiento informado en el tratamiento odontológico - Cléa Adas Saliba Garbin, Artênio José Isper Garbin, Cleide dos Anjos Santos, Patrícia Elaine Gonçalves

físicos, psíquicos, económicos y sociales, además de las posibles alternativas terapéuticas existentes.

Según Diniz y Guilhem(14), el paciente debe decidir en común acuerdo con el profesional sobre sus opciones. Berlinguer y Garrafa(15) señalan que deben respetarse los pensamientos y las opiniones ajenas, resultando en un acuerdo mutuo, lo que se ve reflejado en este estudio, en el cual un $89,4 \%$ de los entrevistados citan que esa decisión deba ser a través de común acuerdo entre profesional y el paciente.

Garbin et al.(16), al entrevistar padres de niños sometidos a tratamiento odontológico en la Facultad de Odontología de Araçatuba/UNESP, observaron que un $65,98 \%$ de ellos no participaron de la decisión del tratamiento. En el presente trabajo, en cambio, el 4,7\% de los cirujanos dentistas entrevistados alegan que esa decisión debe ser tomada sólo por el profesional, puesto que es el que "sabe", y un porcentaje similar opina que la decisión debe ser tomada por el paciente. Con eso, se observó un conflicto entre el posicionamiento del profesional y del paciente sobre quién debe decidir el tratamiento odontológico.

Sobre la participación del paciente y/o responsable legal en la decisión del tratamiento, el 64,8\% de los investigados informa no interferir, concordando con Teixeira(17), según el cual el éxito del mismo sólo es posible con la colaboración del paciente: se basa en la confianza, motivación y en la calidad de la relación. Silva et al.(18) verificaron que el $92,5 \%$ de los cirujanos dentistas entrevistados no consideraban perjudicial discutir cuestiones técnicas con el paciente, posibilitándole el ejercicio de su autonomía. Sin embargo, el 29,4\% de los entrevistados en el presente estudio afirman que la participación del paciente interfiere de manera negativa, pues éste puede no optar por el tratamiento que el cirujano dentista juzga ser el más adecuado, lo que según Kenny(19) puede ocasionar un conflicto en la interacción entre ambos.

Ante eso, Odom et al.(3) mencionan que debe haber comprensión del valor de la relación profesional/paciente, lo que resultaría en un beneficio bilateral y no apenas unilateral, como se observó por largos períodos. Por eso es muy importante que los profesionales de salud tengan conciencia de que el paciente no es más un sujeto pasivo, sino el titular de derechos que, entre otras cosas, le confiere el poder de participar activamente en el tratamiento.
Con relación al lenguaje empleado para explicar el tratamiento al paciente, el 48,2\% alega hacerlo en lenguaje común, concordando con Farah y Ferraro(5) y Chiccone(13), lo que muchas veces no ocurre, como queda en evidencia en el hecho de que el $43,5 \%$ de nuestros entrevistados reconocen que explican el tratamiento en forma técnica. Para Munhoz y Fortes(12), los profesionales de la salud deben presentar las informaciones al paciente con palabras más sencillas, aproximativas, inteligibles y respetuosas, y de forma organizada.

$\mathrm{Al}$ analizar qué documento solicitaban los investigados al paciente antes de empezar el tratamiento, se observó que el $54 \%$ obtenía una autorización y el 15\% no solicitaba nada. Estos últimos están expuestos a situaciones extremamente desfavorables(20). Sólo el $25 \%$ de los entrevistados obtenían el consentimiento informado del paciente y/o de su responsable legal. De acuerdo con Farah y Ferraro(5), el consentimiento del paciente es una condición que debe ser respetada antes de empezar cualquier procedimiento del cirujano dentista. Sin embargo el $2 \%$ de los investigados no respondió, tal vez por desconocer la importancia o el significado del requisito de consentimiento informado.

El problema es que muchas veces el cirujano dentista no tiene conocimiento de la diferencia entre "autorización" y "consentimiento informado", porque la autorización es sólo un documento unilateral; o sea, el paciente autoriza al profesional a ejecutar el servicio, aunque muchas veces sin entenderlo. El consentimiento informado, más allá de ser bilateral, demuestra que el paciente quedó al tanto y es libre para escoger el tipo de tratamiento cuando el caso ofrece más de una alternativa $(21,22)$. De este modo, el documento de consentimiento informado resguarda también al profesional en lo que se refiere al suministro de informaciones suficientes y adecuadas al paciente, de acuerdo con lo señalado en el artículo 14 del Código de Defensa del Consumidor Brasileño(23).

Es necesario que el paciente sea informado sobre las propuestas de tratamiento presentadas(24). Denham (25) menciona que algunos elementos del consentimiento deben ser discutidos con el paciente para que éste otorgue su aprobación, esto es, procedimientos, riesgos, beneficios, alternativas de tratamiento, voluntariedad y confidencialidad. 


\section{Referencias}

1. Goldin JR. Declaraçóes de Helsinque. Sitio en Internet. Disponible en http://www.bioetica.ufrgs.br

2. Clotet J. O consentimento informado nos coiemitês de éticas em pesquisa e na pratica medica: conceituação, origens e atualidades. Revista Bioética 1995; 1(3):7-12.

3. Odom JG, Beemsterboer PL, Pate TD, Haden NK. Revisiting the Status of Dental Ethics Instruction. Journal Dentistry Education 2000; 64(11): 772-774.

4. Ramos DLP. A Proteção do Profissional. In: Feller C, Gorab R. Atualização na clínica odontológica: cursos antagônicos. São Paulo: Artes Medicas; 2000: 581-91.

5. Farah EE, Ferraro L. Como prevenir problemas com os pacientes - responsabilidade civil: para dentistas, médicos eprofissionais da saúde. 3. ed. São Paulo: Quest; 2000.

6. Tanaka H, Garbin CAS. Estudo das reclamaçôes contra cirurgiōes-dentistas no PROCON de Presidente Prudente-SP. Revista Paulista de Odontologia 2003; 25(5): 30-33.

7. Comissão Nacional de Ética Em Pesquisa. Normas para pesquisa envolvendo seres humanos. (Res.CNS 196/96 e outras). Brasília: Ministério da Saúde; 2000.

8. Correia CC, Silva JJ. Pesquisa em seres humanos. Aspectos Bioeticos. Anais Faculdade Odontologia Universidade Federal Pernambuco 1999; 9(1): 57-59.

9. Nunes DS, Fernandes F. Conhecimento, conscientização e atitude do docente no que respeita ao consentimento informado e à autonomia do paciente. Revista da ABENO 2006; 6(1): 11- 19.

10. Dean AG, Dean JA, Burton AH, Dicker RC. Epi Info Version 6.04: a word processing, database, and statistics program for epidemiology on micro-computers. Atlanta, Georgia: Centers for Disease Control; 1990.

11. Corrêa MSNP. Odontopediatria na primeira infância. São Paulo: Santos; 1998.

12. Munôz DR, Fortes PAC. O principio da Autonomia e o Consentimento Livre e Esclarecido. In: Costa SF, Oselka G, Garrafa V, (orgs.) Iniciação a Bioética. Brasília: Conselho Federal de Medicina; 1998: 53-70.

13. Chiccone MV. Informed Consent: imperspective. Nova York: Mid-Atlantic Medical Insurance; 1990.

14. Diniz D, Guilhem D. O que é Bioética? São Paulo: Brasiliense; 2002.

15. Berlinguer G, Garrafa V. O mercado humano: estudo bioético da compra e venda de partes do corpo. Brasília: UnB; 1996.

16. Garbin CAS, Mariano RQ, Machado TP, Garbin AJI. Estudo Bioético das Relaçôes Humanas no tratamento odontológico. Revista Faculdade Odontologia de Lins 2002; 14(1): 54-59.

17. Teixeira R. Entendendo a relação paciente/profissional. Revista Associação Paulista Cirurgiöes Dentista 2000; $54(4)$ : 267-278.

18. Silva KA, Almeida LT, Miranda, IMP, Silveira JLGC. Legislação e ética da relação dentista-paciente. Pesquisa Brasileira Odontopediatria Clinica Integrada 2001; 1(3):31-37.

19. Kenny NP. Bioethics and Canadian Dentristy. Journal Canadian Dental Association 1997; 63(9): 690-694.

20. Goldin JR. O processo de consentimento informado numa perspectiva além da autonomia. In: Universidade do Vale do Itajaí. Pró-Reitoria de pesquisa, pós-graduação, extensão e cultura. Comissão de ética em pesquisa. II seminário sobre ética em pesquisa; 2004: 19-36.

21. Gonçalves CR. A responsabilidade dos médicos, hospitais, dentistas. In: Gonçalves CR. Direito Civil: direito das obrigaçôes. Volume 6, tomo II: responsabilidade civil. 2a ed. São Paulo: Saraiva; 2002: 148-150.

22. Miranda SJ, (coord.) Organização Deocleciano Torrieri Guimarães. Autorização. 2ed. São Paulo: Rideel, 1999. p. 112.

23. Said Cahali Y, (org.) Constituiçâoo Federal, Código Civil, Código de Processo Civil. São Paulo: Editora Revista dos Tribunais, 2003.

24. Weijer C, Dickens B, Meslin EM. Bioethics for clinicians: 10. Research ethics. Canadian Medical Association Journal 1997; 156(8): 1153-1157.

25. Denham JE, Nelson RM. Self-determination is not na Appropriate Model for Understanding Parental Permission and Child Assent. Anesthesia and Analgesia; 2002: 1-3.

Recibido: 9 de junio de 2008

Aceptado: 15 de octubre de 2008 